\title{
A Rigidity Theorem for non-Vacuum Initial Data
}

\author{
Gábor Etesi \\ Yukawa Institute for Theoretical Physics, \\ Kyoto University, \\ 606-8502 Kyoto, Japan \\ etesi@yukawa.kyoto-u.ac.jp
}

\begin{abstract}
In this note we prove a theorem on non-vacuum initial data for general relativity. The result presents a "rigidity phenomenon" for the extrinsic curvature, caused by the nonpositive scalar curvature.

More precisely, we claim that in the case of an asymptotically flat non-vacuum initial data if the spatial metric has everywhere non-positive scalar curvature then the extrinsic curvature cannot be compactly supported.
\end{abstract}

Keywords: Initial value formulation, extrinsic curvature

PACS numbers: 04.20.Ex, 04.20.Ha

\section{Introduction}

According to our experiences there are many different gravitational configurations in our physical world. Therefore if general relativity is a correct theory of gravitational phenomena (at least at low energies) then it is important to know whether or not these various patterns can be modeled in general relativity i.e. Einstein equations provide enough solutions for describing many different gravitational fields. Unfortunately or fortunately Einstein equations form an extraordinary difficult system of nonlinear partial differential equations for the four dimensional Lorentzian metric mainly because of the rich self-interactions of the gravitational field; hence in general it is a hard job to write down explicit solutions in this theory. Therefore all methods which prove at least the existence of solutions are very important. From this viewpoint, the Cauchy problem or initial value formulation of general relativity is maybe the most powerful method to generate plenty of solutions.

As it is well-known, the initial value formulation gives rise to a correspondence between globally hyperbolic space-times and gravitational initial data. Maybe we can say without an exaggeration that the class of globally hyperbolic space-times is the most important class of space-times from the physical point of view. Consequently the initial data formulation provides not only many but also physically relevant solutions. The constraint equations between initial data are in the focal point of the initial data formulation. The question is whether or not these constraint equations are easier to solve than the original Einstein equation itself making the method effective. Of course, the answer is typically yes.

This motivates the serious efforts made in order to understand the structure and provide solutions of constraint equations. Far from being complete we just mention the early works of Lichnerowicz [15], Choquet-Bruhat et al. [2][3][6] , Fisher-Marsden [8], Christodoulou-Kleinerman[7]. These works mainly deal with the analytical properties of the solutions. Witt proved the existence of solutions on a general three-manifold [19]. More recently, in a sequence of papers 
Isenberg, Moncrief, Choquet-Bruhat and York proved the existence of solutions under milder and milder assumptions, cf. e.g. [12][4][13][5].

The constraint equations involve the scalar curvature of the metric on the underlying Cauchy surface which is a three dimensional smooth manifold. Various properties of the solutions depend crucially on the scalar curvature, especially on its sign. But we know that in the problem of describing the sign of the scalar curvature, especially on a compact manifold, one encounters with the topology of the space. Parallelly to the investigations of solutions of the constraint equations by physicist and mathematicians, mathematicians proved remarkable results on the properties of the scalar curvature of Riemannian manifolds. By an early general result of Kazdan and Warner [14] we know that for compact manifolds of dimension greater than two there is no constraint on the scalar curvature if there is at least one point where it is negative. This shows that it is easy to construct manifolds with negative scalar curvature. If we wish to construct manifolds with non-negative scalar curvature, however, we have to face various obstacles coming from the topology of the manifold. We just mention two basic examples. By results of Lichnerowicz and Hitchin, on spin manifolds it is often impossible to construct metrics of positive scalar curvature because of a subtle topological invariant, the so-called $\hat{A}$-genus [11][16]. Moreover, in three dimensions, the size of the fundamental group provides another obstruction for positive scalar curvature by results of Gromow-Lawson [9] and Schoen-Yau [17]. An excellent survey on this branch of differential geometry is [1].

These observations make it not surprising that the topology of the Cauchy surface has a strong influence on the properties of initial data on it. The goal of this paper is to understand this link a bit better. Our motivation is a paper by Witt [19] who studied the problem of existence of initial data on general three manifolds and examined certain characteristics of these initial data. In Section 3 we prove a theorem which states that on open Riemannian manifolds with everywhere non-positive scalar curvature the extrinsic curvature field of a non-vacuum initial data cannot be compactly supported i.e. it has a "tail" at infinity although this tail may have sufficiently fast fall-off to make such an initial data still asymptotically flat. The proof of this theorem is elementary and is based on the following idea.

By using the initial data set $(M, g, k)$ and the assumption that supp $k$ is compact, we construct another "universal" initial data set $(M, g, \varphi g)$ where $\varphi: M \rightarrow \mathbb{R}$ is a compactly supported at least once continuously differentiable (or $C^{1}$ ) function on $M$ (with a little more effort this function could be smoothened but we do not need this). However this leads us to a contradiction if the scalar curvature of $g$ is non-positive everywhere. In other words, we deform the original initial data set into a standard one whose properties are easier to understand.

Acknowledgement. The author thanks Prof. H. Kodama (YITP, Japan) and Prof. L.B. Szabados (KFKI, Hungary) for the fruitful discussions and to the unknown referee of JMP for calling our attention to some weak points in the original version of the proof. The work was supported by JSPS grant no. P99736.

\section{Background material}

First let us introduce some notations. Let $W$ be a smooth manifold. We will call a tensor field $T$ of type $(m, n)$ over $W$ if it is a smooth section of the bundle

$$
T^{(m, n)} W:=\underbrace{T W \otimes \ldots \otimes T W}_{m} \otimes \underbrace{T^{*} W \otimes \ldots \otimes T^{*} W}_{n} .
$$


Remember that an initial data set for general relativity is a triple $(M, g, k)$, where $M$ is a (not necessarily compact) connected, oriented, smooth three-manifold, $g=\left(g_{i j}\right)$ is a smooth, complete Riemannian metric on $M$ i.e. a non-degenerate smooth symmetric tensor field of type $(0,2)$ on $M$ while $k=\left(k_{i j}\right)$ is a smooth, symmetric tensor field on $M$ also of $(0,2)$-type. These fields must satisfy the following constraint equations [10][18]:

$$
\left\{\begin{array}{l}
s_{g}-|k|_{g}^{2}+\operatorname{tr}^{2} k=16 \pi \rho, \\
\operatorname{div}(k-(\operatorname{tr} k) g)=8 \pi J, \\
\rho \geq|J|_{g} \geq 0 .
\end{array}\right.
$$

Here $s_{g}$ is the scalar curvature of the metric $g$ and $|\cdot|_{g}$ denotes various norms given by the induced scalar product on $T^{(m, n)} M$, e.g. $|k|_{g}^{2}=\langle k, k\rangle=k_{i j} k^{i j}$. The operator $\operatorname{tr}: T^{(m, n)} M \rightarrow T^{(m-1, n-1)} M$ is the trace with respect to the metric, e.g. $\operatorname{tr} k=k_{i}^{i}$. For the sake of simplicity in the second equation we also denote by $g$ and $k$ the $(1,1)$-tensors with respect to the metric $g$ (i.e. $g=$ $\left(g_{j}^{i}\right), k=\left(k_{j}^{i}\right)$ in the second equation). The linear differential operator $\operatorname{div}: C^{\infty}\left(T^{(m, n)} M\right) \rightarrow$ $C^{\infty}\left(T^{(m-1, n)} M\right)$ is the covariant divergence, defined by

$$
\operatorname{div} T:=\operatorname{tr}(\nabla T)
$$

where $T$ is a tensor field of $(m, n)$-type and $\nabla$ is the Levi-Civitá covariant derivative of the metric $g$. The smooth function $\rho: M \rightarrow \mathbb{R}$ is the energy-density, and the smooth covector field $J \in C^{\infty}\left(T^{*} M\right)$ with $|J|_{g}^{2}=\langle J, J\rangle=J_{i} J^{i}$ is interpreted as the momentum-density of matter.

Supposing the energy- and momentum-densities correspond to classical non-dissipative matter sources or vacuum $(J=0, \rho=0)$, the coupled Einstein-equations can be used to evolve the initial data set $(M, g, k)$ into a (globally hyperbolic) smooth space-time $(N, h)$ where $N \cong M \times \mathbb{R}$ and $M$ is a Cauchy surface in $N$; furthermore $\left.h\right|_{M}=g$ and $k$ is the second fundamental form or extrinsic curvature of $M$ in $(N, h)$ [10] [18].

Also remember that the open oriented three-manifold $M$ has an end $E \subset M$ if there is a compact set $C \subset M$ such that $M \backslash C=E$ and $E \cong S_{g} \times\left(\mathbb{R}^{+} \backslash\{0\}\right)$ where $S_{g}$ is a compact, oriented surface of genus $g$ and $\mathbb{R}^{+}=[0, \infty)$. An initial value data set $(M, g, k)$ is called asymptotically flat along $E$ if $M$ has an end $E \cong S^{2} \times\left(\mathbb{R}^{+} \backslash\{0\}\right)$ and the following asymptotical fall-off conditions hold for the complete metric $g$ and the field $k\left(r\right.$ parameterizes $\mathbb{R}^{+}$in $\left.E\right)$ :

$$
\begin{array}{ll}
\left(\left.g\right|_{E}\right)_{i j}=\delta_{i j}+O(1 / r), & \left(\left.k\right|_{E}\right)_{i j}=O\left(1 / r^{2}\right), \\
\partial_{l}\left(\left.g\right|_{E}\right)_{i j}=O\left(1 / r^{2}\right), & \partial_{l}\left(\left.k\right|_{E}\right)_{i j}=O\left(1 / r^{3}\right), \\
\partial_{l} \partial_{k}\left(\left.g\right|_{E}\right)_{i j}=O\left(1 / r^{3}\right) . &
\end{array}
$$

Notice that the definition of a manifold with an end does not exclude the possibility that $M$ still has a boundary, different from the end $E$ (strictly speaking, $E$ is not a boundary). But the boundary points are added to $M$ because $C=M \backslash E$ is compact according to our assumption. Consequently geodesic completeness of $g$ requires that this extra boundary must be empty, in other words $M$ is diffeomorphic to the punctured manifold $\widetilde{M} \backslash\{y\}$ where $\widetilde{M}$ is a connected, compact, oriented three-manifold without boundary.

Finally, the support of a tensor field $T \in C^{\infty}\left(T^{(m, n)} W\right)$ is the closed set

$$
\operatorname{supp} T:=\overline{\{x \in W \mid T(x) \neq 0\}} \text {. }
$$


After this background material, we are in a position to prove our theorem. The motivation is the following.

\section{The theorem}

It was proved by Witt [19] that every three-manifold with an end admits a non-vacuum, asymptotically flat initial data set. For a typical three-manifold, the resulting Cauchy developed spacetime does not admit maximal slices, however; i.e. there are no maximal space-like submanifolds whose extrinsic curvature is identically zero. One may raise the question: in what extent are these slices not maximal? In other words what are the conditions on a Riemannian manifold $(M, g)$ for its extrinsic curvature in the Cauchy development to be compactly supported at least? We will answer this question in our theorem.

Theorem (Rigidity for non-vacuum initial data). Let $(M, g)$ be a connected, oriented, complete Riemannian three-manifold with an end $E \cong S^{2} \times\left(\mathbb{R}^{+} \backslash\{0\}\right)$. Suppose the scalar curvature $s_{g}$ of $g$ is non-positive everywhere and there is a non-vacuum initial data set $(M, g, k)$ on it which is asymptotically flat along the end $E$. Then $\operatorname{supp} k$ is non-compact.

Proof. Since the scalar curvature is non-positive, the set supp $s_{g}$ consists of the closure of those points where $s_{g}$ is negative. Then the first and third (in)equalities of (1) show that $\operatorname{supp} s_{g} \subseteq \operatorname{supp} k$ therefore if the scalar curvature is negative everywhere the statement is trivially true, consequently we may assume that supp $s_{g} \subset M$. In the same fashion, since $(M, g, k)$ is a non-vacuum data set, there is a point $x_{0} \in M$ such that $\rho\left(x_{0}\right) \neq 0$. This yields $\operatorname{supp} \rho \neq \emptyset$. Being the scalar curvature non-positive, via the first and third (in)equalities of (1) again we have $k\left(x_{0}\right) \neq 0$ i.e., supp $\rho \subseteq \operatorname{supp} k$. Therefore if the energy density is supported everywhere the theorem is again trivially valid consequently we may assume supp $\rho \subset M$. Consider a subset $C \subset M$ such that supp $s_{g} \subset C$ and supp $\rho \subset C$ and suppose the decomposition $M=C \cup E$ is valid where $E$ denotes the end of $M$. Consequently by the structure of $M$ we may assume that $C$ is compact. This shows that there is a constant

$$
0<a:=\sup _{x \in C}\left(-|k(x)|_{g}^{2}+\operatorname{tr}^{2} k(x)\right)<\infty .
$$

Consider a triple $(M, g, \varphi g)$ where $\varphi: M \rightarrow \mathbb{R}$ is a $C^{1}$-function. This triple is a weak initial data set if it obeys the constraint equations (here by "weak" we mean that the initial data set in question is not smooth, only $C^{k}$ for some $k \in \mathbb{N}$ ):

$$
\left\{\begin{array}{l}
s_{g}+6 \varphi^{2}=16 \pi \rho, \\
-2 \operatorname{div}(\varphi g)=-2 \operatorname{tr}(\nabla(\varphi g))=-2 \operatorname{tr}(\mathrm{d} \varphi \otimes g)=8 \pi J \\
\rho \geq|J|_{g} \geq 0 .
\end{array}\right.
$$

In the second equation we have used the fact that $\nabla g=0$. These (in)equalities can be combined into a first order partial differential inequality for the unknown function $\varphi$ :

$$
\frac{1}{4}\left(s_{g}+6 \varphi^{2}\right) \geq|\mathrm{d} \varphi|_{g}
$$

taking into account that $|\operatorname{tr}(\mathrm{d} \varphi \otimes g)|_{g}=|\mathrm{d} \varphi|_{g}$. Assume $\emptyset \neq \operatorname{supp} k \subset M$ is compact i.e., the theorem is not true. In this case we construct a compactly supported function $\varphi$, out of the 
original data $(M, g, k)$ such that $(M, g, \varphi g)$ is a weak initial data set. We achieve this in three steps.

(i) Construction of $\varphi$ in the compact interior of $M$. Let us identify the end $E \subset M$ with $S^{2} \times\left(\mathbb{R}^{+} \backslash\{0\}\right)$. By assumption supp $k$ is compact in $M$ consequently there is an $R_{1} \in \mathbb{R}^{+}$ satisfying $S^{2} \times\left(R_{1}, \infty\right) \not \subset \operatorname{supp} k$. Note that this is possible only if $\left.s_{g}\right|_{S^{2} \times\left(R_{1}, \infty\right)}=0$. We can take the choice $C:=M \backslash\left(S^{2} \times\left(R_{1}, \infty\right)\right)$ for the compact set used in the definition of the constant $a$. We construct the function $\varphi$ in $C$ as follows:

$$
\varphi(x):=-\sqrt{a}, \quad x \in C .
$$

In other words $\varphi$ is a constant negative function on $M$ except the infinite tube $S^{2} \times\left(R_{1}, \infty\right)$. Note that with this function (2) is trivially satisfied in $C$ because $(M, g, k)$ is an initial data set on $C$.

(ii) Construction of $\varphi$ along an annulus in $E$. Consider an inner point $x_{0} \in C \subset M$ where $\rho\left(x_{0}\right)>0$ and $k\left(x_{0}\right) \neq 0$. There is an open (geodesic) ball $B_{\varepsilon}\left(x_{0}\right) \subset M$ of radius $\varepsilon>0$ such that $\left.\rho\right|_{B_{\varepsilon}\left(x_{0}\right)}>0$ and $\left.k\right|_{B_{\varepsilon}\left(x_{0}\right)} \neq 0$. Consider the annulus $U_{\varepsilon}:=\overline{B_{\varepsilon}\left(x_{0}\right) \backslash B_{\frac{\varepsilon}{2}}\left(x_{0}\right)} \cong S^{2} \times\left[\frac{\varepsilon}{2}, \varepsilon\right]$. Take another constant $R_{1}<R_{2}<\infty$ and the diffeomorphism

$$
\beta: U_{\varepsilon} \longrightarrow S^{2} \times\left[R_{1}, R_{2}\right], \quad x_{t}=(p, t) \longmapsto\left(p, R_{1}+\frac{2 t-\varepsilon}{\varepsilon}\left(R_{2}-R_{1}\right)\right)=(p, r)
$$

where $p \in S^{2}$ and the point $x_{t} \in U_{\varepsilon}$ is identified with $(p, t) \in S^{2} \times\left[\frac{\varepsilon}{2}, \varepsilon\right]$. Here $S^{2} \times\left[R_{1}, R_{2}\right]$ is also an annulus in the tube $E$. By assumption $g$ is asymptotically flat i.e., the function $\sqrt{g^{11}} \geq 0$ is bounded consequently there is a constant

$$
0<b:=\sup _{x \in M} \sqrt{g^{11}(x)}<\infty
$$

(here $x^{1}=r$ ). Choose a smooth function $\psi:\left[R_{1}, R_{2}\right] \rightarrow \mathbb{R}^{-}$. Viewing it as a function on $S^{2} \times\left[R_{1}, R_{2}\right]$ (i.e. a function depending only on $r$ ), one obtains the estimate

$$
b\left|\psi^{\prime}\right| \geq\left|\sqrt{g^{11}} \psi^{\prime}\right|=|\mathrm{d} \psi|_{g}
$$

where prime denotes differentiation with respect to $r$. Now we define $\psi$ as follows:

$$
\psi\left(\beta\left(x_{t}\right)\right):= \begin{cases}-\sqrt{a} & \text { if } t=\frac{\varepsilon}{2} \\ \text { arbitrary but the derivative of } \psi \text { is small } & \text { if } t \in\left(\frac{\varepsilon}{2}, \varepsilon\right) \\ 0 & \text { if } t=\varepsilon\end{cases}
$$

In this definition the smallness of $\psi^{\prime}$ means the following. Consider a differentiable curve $\gamma$ : $\left[\frac{\varepsilon}{2}, \varepsilon\right] \rightarrow U_{\varepsilon}$ given by

$$
t \longmapsto x_{t}:=\left(\Theta_{\frac{\varepsilon}{2}}+A \sin \left(R_{2}-R_{1}\right) t, \phi_{\frac{\varepsilon}{2}}+A \sin \left(R_{2}-R_{1}\right) t, t\right) .
$$

This is a high-speed curve because it oscillates rapidly inside $B_{\varepsilon}\left(x_{0}\right)$. More precisely, for its speed $|\dot{\gamma}(t)|_{g} \sim R_{2}-R_{1}$ is valid (dot denotes differentiation with respect to $t$ ). We can take a choice for the point $x_{0}$ and the amplitude $A$, the initial phases $\Theta_{\frac{\varepsilon}{2}}$ and $\phi_{\frac{\varepsilon}{2}}$ of the curve $\gamma$ such that

$$
\mathrm{d}\left(\sqrt{-\left|k\left(x_{t}\right)\right|_{g}^{2}+\operatorname{tr}^{2} k\left(x_{t}\right)}\right)(\dot{\gamma}(t)) \sim-\left(R_{2}-R_{1}\right)<0
$$


holds for each $t \in\left[\frac{\varepsilon}{2}, \varepsilon\right]$. Then we suppose

$$
0 \leq \psi^{\prime}\left(\beta\left(x_{t}\right)\right) \leq \min \left(-\frac{\varepsilon}{4\left(R_{2}-R_{1}\right)} \mathrm{d}\left(\sqrt{-\left|k\left(x_{t}\right)\right|_{g}^{2}+\operatorname{tr}^{2} k\left(x_{t}\right)}\right)(\dot{\gamma}(t)), \frac{16 \pi}{b} \rho\left(x_{t}\right)\right) .
$$

It is also clear that such a function exists if $R_{2}$ is suitable large: let $\psi$ be an arbitrary smooth, negative-valued function $\psi: S^{2} \times\left[R_{1}, R_{2}\right] \rightarrow \mathbb{R}^{-}$with initial value $\psi\left(p, R_{1}\right)=\psi\left(\beta\left(x_{\frac{\varepsilon}{2}}\right)\right)=-\sqrt{a}$. Suppose there is an interval $[R, R+T] \subset\left[R_{1}, R_{2}\right]$ such that $\psi^{\prime}$ obeys (4) but there is a constant $c>0$ with $\psi^{\prime}(p, r) \geq c$ if $r \in[R, R+T]$. This constant can be chosen to be independent of $R_{2}-R_{1}$. In this case we can estimate for large $R_{1}$ and $R_{2}$ as follows:

$$
\psi\left(p, R_{2}\right) \geq-\sqrt{a}+\frac{1}{2} \int_{R_{1}}^{R_{2}} \psi^{\prime}(p, r) \mathrm{d} r \geq-\sqrt{a}+\frac{1}{2} \int_{R}^{R+T} \psi^{\prime}(p, r) \mathrm{d} r \geq-\sqrt{a}+\frac{c}{2} T .
$$

In other words if $T$ that is, $R_{2}-R_{1}$ is sufficiently large we can achieve that $\psi\left(p, R_{2}\right)=0$. We choose $\varphi$ on $S^{2} \times\left[R_{1}, R_{2}\right]$ to be the $\psi$ just constructed.

It is not difficult to check that $\varphi$ obeys (2) in $S^{2} \times\left[R_{1}, R_{2}\right]$. Indeed, by the definition of the constant $a$ we have

$$
\varphi\left(p, R_{1}\right)=\varphi\left(\beta\left(x_{\frac{\varepsilon}{2}}\right)\right)=-\sqrt{a} \leq-\sqrt{-\left|k\left(x_{\frac{\varepsilon}{2}}\right)\right|_{g}^{2}+\operatorname{tr}^{2} k\left(x_{\frac{\varepsilon}{2}}\right)} .
$$

Taking suitable large $R_{1}$ and $R_{2}$, exploiting the decay of the metric $g$ and using (4) this implies that for each $t \in\left[\frac{\varepsilon}{2}, \varepsilon\right]$ we have

$$
\begin{gathered}
\varphi\left(\beta\left(x_{t}\right)\right)=\varphi(p, r)=-\sqrt{a}+\int_{R_{1}}^{r} \mathrm{~d} \varphi(p, \varrho)\left(\beta^{\prime}\left(x_{\tau}\right)\right) \mathrm{d} \varrho= \\
-\sqrt{a}+\int_{R_{1}}^{r} \varphi^{\prime}(p, \varrho)\left(g_{11}(p, \varrho)+A \frac{\varepsilon}{2}\left(g_{12}(p, \varrho)+g_{13}(p, \varrho)\right) \cos \frac{\varepsilon}{2}\left(\varrho+R_{2}\right)\right) \mathrm{d} \varrho \leq \\
-\sqrt{a}+2 \int_{R_{1}}^{r} \varphi^{\prime}(p, \varrho) \mathrm{d} \varrho=-\sqrt{a}+\frac{4\left(R_{2}-R_{1}\right)}{\varepsilon} \int_{\frac{\varepsilon}{2}}^{t} \varphi^{\prime}\left(\beta\left(x_{\tau}\right)\right) \mathrm{d} \tau \leq \\
-\sqrt{-\left|k\left(x_{\frac{\varepsilon}{2}}\right)\right|_{g}^{2}+\operatorname{tr}^{2} k\left(x_{\frac{\varepsilon}{2}}\right)}-\int_{\frac{\varepsilon}{2}}^{t} \mathrm{~d}\left(\sqrt{-\left|k\left(x_{\tau}\right)\right|_{g}^{2}+\operatorname{tr}^{2} k\left(x_{\tau}\right)}\right)(\dot{\gamma}(\tau)) \mathrm{d} \tau=-\sqrt{-\left|k\left(x_{t}\right)\right|_{g}^{2}+\operatorname{tr}^{2} k\left(x_{t}\right)} .
\end{gathered}
$$

Consequently

$$
\varphi^{2}\left(\beta\left(x_{t}\right)\right) \geq-\left|k\left(x_{t}\right)\right|_{g}^{2}+\operatorname{tr}^{2} k\left(x_{t}\right)
$$

Therefore, since $s_{g}\left(\beta\left(x_{t}\right)\right)=0$ and $0 \geq s_{g}\left(x_{t}\right)$, we can write

$$
\frac{1}{4}\left(s_{g}\left(\beta\left(x_{t}\right)\right)+6 \varphi^{2}\left(\beta\left(x_{t}\right)\right)\right)=\frac{3}{2} \varphi^{2}\left(\beta\left(x_{t}\right)\right) \geq s_{g}\left(x_{t}\right)-\left|k\left(x_{t}\right)\right|_{g}^{2}+\operatorname{tr}^{2} k\left(x_{t}\right)=16 \pi \rho\left(x_{t}\right) .
$$

Moreover, also by (4), we have for the same $x_{t} \in U_{\varepsilon}$ that $16 \pi \rho\left(x_{t}\right) \geq b \varphi^{\prime}\left(\beta\left(x_{t}\right)\right)$. This gives rise to our key inequality

$$
\frac{3}{2} \varphi^{2}\left(\beta\left(x_{t}\right)\right) \geq b \varphi^{\prime}\left(\beta\left(x_{t}\right)\right)
$$


showing via (3) that (2) is again satisfied in the annulus $S^{2} \times\left[R_{1}, R_{2}\right]$.

(iii) Construction of $\varphi$ along the remaining part of the infinitely long tube in $M$. Finally, define

$$
\varphi(x):=0 \quad \text { if } x \in S^{2} \times\left[R_{2}, \infty\right) .
$$

Again, (2) is trivially valid.

Consider the function $\varphi: M \rightarrow \mathbb{R}^{-}$defined through (i)-(iii). This is a continuous negative function on $M$ and is compactly supported: it is equal to zero for all $r \geq R_{2}$ and equal to the constant $-\sqrt{a}$ if $r \leq R_{1}$. Its derivative is also compactly supported in $S^{2} \times\left[R_{1}, R_{2}\right]$ and is positive. Moreover $\varphi$ can be adjusted to be $C^{1}$ on $M$ (note that $\varphi$ is smooth except the junction points): it is clearly $C^{1}$ at $r=R_{2}$ by (5). However, by exploiting the freedom in the construction of $\varphi$ in the inner points of the annulus, we can deform it to be $C^{1}$ at $r=R_{1}$ as well (i.e., we may assume that $\varphi^{\prime}(p, r) \rightarrow 0$ as $\left.r \rightarrow R_{1}\right)$. In this way we have constructed a weak $C^{1}$ initial data set $(M, g, \varphi g)$ (with a little effort we could smooth this data but we do not need this).

The compactly supported $\varphi$ depends nontrivially only on $r$ with $(p, r)=\beta\left(x_{t}\right) \in S^{2} \times\left[R_{1}, R_{2}\right]$ and satisfies the ordinary differential inequality (5). Now we demonstrate that it is impossible. Dividing by $\varphi^{\prime 2}$ and taking reciprocies in (5) we get

$$
\left(\frac{\varphi^{\prime}}{\varphi}\right)^{2} \leq \frac{3 \varphi^{\prime}}{2 b}
$$

which is nothing but

$$
-\sqrt{\frac{3 \varphi^{\prime}}{2 b}} \leq \frac{\varphi^{\prime}}{\varphi} \leq \sqrt{\frac{3 \varphi^{\prime}}{2 b}} .
$$

By integrating the left inequality from $R_{1}$ to $r<R_{2}$ we arrive at the following estimate:

$$
\log \sqrt{a}-\sqrt{\frac{3}{2 b}} \int_{R_{1}}^{R_{2}} \sqrt{\varphi^{\prime}(p, \varrho)} \mathrm{d} \varrho \leq \log (-\varphi(p, r)) .
$$

At this point we have used the inequality

$$
0<\int_{R_{1}}^{r} \sqrt{\varphi^{\prime}(p, \varrho)} \mathrm{d} \varrho \leq \int_{R_{1}}^{R_{2}} \sqrt{\varphi^{\prime}(p, \varrho)} \mathrm{d} \varrho<\infty
$$

for the non-negaitve function $\varphi^{\prime}$. This shows that the logarithm of $\varphi$ is bounded from below. However being $\varphi$ compactly supported, $\log (-\varphi(p, r))$ is unbounded, as $r$ approaches $R_{2}$. Consequently the last but one inequality shows a contradiction yielding our original assumption, that $\operatorname{supp} k$ is compact, was wrong. We finished the proof. $\diamond$

Remarks. 1.We would like to summarize here how the original initial data $(M, g, k)$ was used in the construction because apparently its behaviour has been taken into account only in a particular small ball $B_{\varepsilon}\left(x_{0}\right)$. But in fact the construction is sensitive for the global characteristics of the original initial data. In step (i) we considered $(M, g, k)$ in the whole interior $C$ by exploiting the existence of the constant $a$ which is in some sense the maximum of $k$ in the whole compact $C$. This enabled us to "pump up" the original initial data in $C$ into a standard one which corresponds to the extremal point(s) of the original extrinsic curvature in some sense. Concerning part (iii), we have seen in the beginning of the proof that the only interesting possibility for our would-be 
initial data with compactly supported extrinsic curvature was the case where both the scalar curvature and energy-density were compactly supported. Consequently all fields in the initial data vanish along the tube for very large $r$ yielding the hypothetical initial data did not carry "information" along an infinitely long part of the end $E$. This is in accordance with the fact that our adjusted universal initial data $(M, g, \varphi g)$ was also trivial on this portion. Finally, part (ii) which is the descending regime, is nothing but a magnification of the behaviour of $(M, g, k)$ in a small ball where matter is present via the diffeomorphism $\beta$. Indeed this small ball is responsible for the details of the fall-off of $\varphi$ (we could have used equally well any other ball) however the fact that this function can vanish within a finite distance, is again guaranteed by the global properties of the original would-be initial data set: namely the only interesting case was when all fields were compactly supported.

2. Note that even if $\operatorname{supp} k$ is non-compact the non-vacuum data $(M, g, k)$ may be asymptotically flat, as it is shown by Witt [19] who constructs non-vacuum, asymptotically flat initial data for every three-manifold with an end. But the above theorem is sharp in the following sense. If we allow for a Riemannian manifold $(M, g)$ to have positive scalar curvature in a suitable region in $M$, it is possible to construct non-vacuum asymptotically flat initial data with compactly supported second fundamental form. An example is the Tolman-Bondi solution. This is because in this case the key inequality (5) can be written in the form

$$
\frac{1}{4}\left(s_{g}+6 \varphi^{2}\right) \geq b \varphi^{\prime}
$$

with $s_{g}>0$ in the positive scalar curvature regime and it may have compactly supported solutions. But if $s_{g}$ is still negative somewhere, then $k$ is non-zero in that point, consequently the initial surface is not a maximal slice in this case.

3. Notice that the above considerations do not remain valid for vacuum initial data. For example, the Schwarzschild space-time has initial data with non-positive scalar curvature (namely it is identically zero) but the extrinsic curvature of the initial surface is compactly supported (namely identically zero i.e., the initial surface is a maximal slice). We conjecture that the analogue of the above theorem for vacuum initial data is the following: if $(M, g)$ is an asymptotically flat three-manifold with somewhere negative scalar curvature, then any vacuum initial data set $(M, g, k)$ cannot be asymptotically flat (i.e., the extrinsic curvature cannot decay at the required rate). But in this moment we are unable to prove this.

\section{Concluding Remarks}

In the previous section we have studied gravitational initial data from a general point of view. We have found that in the case of non-positive scalar curvature, the behaviour of the extrinsic curvature becomes very "rigid": for open manifolds, the fall-off of the extrinsic curvature cannot be arbitrary.

The negativity of the scalar curvature becomes important by an early general result of Kazdan and Warner[14]:

Theorem (Kazdan-Warner). Let $W$ be a compact manifold with $\operatorname{dim} W \geq 3$, and $f: W \rightarrow \mathbb{R}$ be a smooth function on it such that there is a point $x \in W$ obeying $f(x)<0$. Then there is a smooth Riemannian metric $h$ on $W$ such that $s_{h}=f$ i.e. whose scalar curvature is the prescribed function $f . \diamond$ 
The theorem demonstrates that a compact manifold of sufficiently large dimension always can be endowed with a metric with somewhere negative scalar curvature. This shows, taking into account the constraint equations, that it is relatively easy to construct initial data with somewhere nonzero extrinsic curvature.

The classical results of Gromow-Lawson [9] and Schoen-Yau [17], however, show that closed three-manifolds whose prime decomposition contains a $K(\pi, 1)$ factor (this implies such manifolds have infinite fundamental groups) do not carry any metric with positive scalar curvature. Consequently initial data with positive scalar curvature must be rare at least in the compact case.

If the compact $\widetilde{M}$ does not have positive scalar curvature, the punctured, open manifold $M=\widetilde{M} \backslash\{y\}$ of this type, which is nothing but a manifold with an end, does not have a metric with non-negative scalar curvature, too. Consequently these punctured manifolds do not admit non-vacuum, asymptotically flat initial data with identically zero extrinsic curvature. Furthermore if the scalar curvature is everywhere non-positive then this extrinsic curvature has non-compact support, as we have seen.

These results are quite surprising because all the fields in question are defined in the class of smooth functions so one would expect that initial data can be altered locally in a non-trivial way. In other words we have reduced the local degrees of freedom of the gravitational field in some sense.

\section{References}

[1] Besse, A.: Einstein Manifolds, Springer-Verlag, Berlin (1987);

[2] Bruhat, Y.: The Cauchy Problem, in: Gravitation (ed.: L. Witten), 130-168, John Wiley \& Sons, Inc., New York, London (1962);

[3] Choquet-Bruhat, Y., Geroch, R.P.: Global Aspects of the Cauchy Problem in General Relativity, Commun. Math. Phys. 14, 329-335 (1969);

[4] Choquet-Bruhat, Y., Isenberg, J., Moncrief, V.: Solution of constraints for Einstein equations, C.R. Acad. Sci. Paris, Sér. I 315, 349-355 (1992);

[5] Choquet-Bruhat, Y., Isenberg, J., York Jr., J.W.: Einstein constraints on asymptotically Euclidean manifolds, Phys. Rev. D61, 084034-1-084034-20 (2000);

[6] Choquet-Bruhat, Y., York Jr., J. W.: The Cauchy Problem, in: General Relativity and Gravitation (ed.: A. Held), Vol. 1, 99-172, Plenum Press, New York (1980);

[7] Christodoulou, D., Klainerman, S.: The Global Nonlinear Stability of the Minkowski SpaceTime, Princeton Univ. Press, Princeton, New Jersey (1993);

[8] Fisher, A.E., Marsden, J.E.: Initial Value Problem and the Dynamical Formulation of General Relativity, in: General Relativity (ed.: S.W. Hawking and W. Israel), 138-211, Cambridge University Press, Cambridge (1979);

[9] Gromow, M., Lawson, H.B.: Positive Scalar Curvature and the Dirac Operator on Complete Riemannian Manifolds, Publ. Math. IHES 58, 83-196 (1983); 
[10] Hawking, S.W., Ellis, G.F.R.: The Large Scale Structure of Space-Time, Cambridge University Press, Cambridge (1973);

[11] Hitchin, N.: Harmonic Spinors, Adv. Math. 14, 1-55 (1974);

[12] Isenberg, J.: Constant mean curvature solutions of the Einstein constraints on closed manifolds, Class. Quant. Grav. 12, 2249-2274 (1995);

[13] Isenberg, J., Moncrief, V.: A set of constant mean curvature solutions of the Einstein constraint equations on closed manifolds, Class. Quant. Grav. 13, 1819-1842 (1996);

[14] Kazdan, J.L., Warner, F.W.: Scalar Curvature and Conformal Deformation of Riemannian Structure, Journ. Diff. Geom. 10, 113-134 (1975);

[15] Lichnerowicz, A.: L'integration des Equations de la Gravitation Relativiste et le Probleme des $n$ Corps, J. Math. Pures Appl. 23 37-63 (1944);

[16] Lichnerowicz, A.: Spineurs Harmoniques C.R. Acad. Sci. Paris 257, 7-9 (1963);

[17] Schoen, R.: Minimal Surfaces and Positive Scalar Curvature, in: Proceedings of the International Congress of Mathematics (Warszawa, 1983), pp. 575-578, PWN, Warszawa (1984);

[18] Wald, R.M.: General Relativity, University of Chicago Press, Chicago (1984);

[19] Witt, D.M.: Vacuum Spacetimes that Admit no Maximal Slice, Phys. Rev. Lett. 57, 13861389 (1986). 